\title{
Improving Patient Flow Using Lean Methodology: an Emergency Medicine Experience
}

Lori Rutman, $M D, M P H^{1,2, *}$

Kimberly Stone, $M D^{1,2}$

Jennifer Reid, $M D^{1,2}$

George A. (Tony) Woodward, MD, MBA ${ }^{1,2}$

Russell Migita, $M D^{1,2}$

\author{
Address \\ ${ }^{1}$ University of Washington School of Medicine, Seattle, WA, USA \\ *,2Division of Pediatric Emergency Medicine, Seattle Children's Hospital, \\ 4800 Sand Point Way NE, Seattle, WA, 98105, USA \\ Email: lori.rutman@seattlechildrens.org
}

Published online: 28 0ctober 2015

C) Springer International Publishing AG 2015

This article is part of the Topical Collection on Quality Improvement

Keywords Lean - Quality improvement $\cdot$ Patient flow $\cdot$ Emergency medicine $\cdot$ Pediatrics $\cdot$ Continuous performance improvement $\cdot$ Process $\cdot$ Waste $\cdot$ Value

\section{Opinion statement}

In today's rapidly changing health care milieu, organizations are expected to continuously improve the quality of care delivered to an expanding population of patients. To do so, they need a framework for developing, testing and implementing changes. Lean provides a methodology to engage workers and leaders to identify waste in a process, develop standards, implement a change, assess the results of that change, review next steps, and repeat the process. This can be successfully accomplished in the highly variable world of emergency medicine and can help health care providers be more productive, engaged, and satisfied while enabling patients to receive the value-added care they want and expect. Successful implementation of Lean or any other improvement framework requires that the hospital and medical leadership are all strong supporters of the methodology, speak the same process improvement language and are able to generate support and resources for operation-wide forward movement. 


\title{
Introduction
}

Lean methodology is derived from principles of the Toyota Production System, developed in the 1950s by Taiichi Ohno [1], who took inspiration from the work of W. Edwards Deming [2], Henry Ford's moving assembly lines, and United States (US) supermarkets. Lean involves principles, methods, and tools to understand and improve the performance of a system. Key principles include the elimination of unnecessary waste, minimizing delays, just-in-time delivery of products and services, worker empowerment, and continuous improvement. Examples of Lean tools and methods include value stream mapping, rapid cycle improvement sessions (kaizen), process standardization (standard work), 5S (a method for organizing and standardizing workspaces), physical layout improvements that minimize travel time, and mistake-proofing/failure prevention [3]. Lean was first applied in the US automobile industry and has subsequently spread to other manufacturers [4] as well as the UK National Health Service [5] with much success. When the Institute of Medicine (IOM) published Crossing the Quality Chasm: A New Health System for the 21st Century in 2012, the authors described the current US health care system as highly fragmented, unnecessarily redundant, and prone to long wait times. The report emphasized the need to redesign the system to improve health care quality and patient safety [6]. As a result, there has been a push for further expansion of Lean methodologies into the US health care milieu [7-14]. Health care systems such as Virginia Mason in Seattle, WA [9, 15] and ThedaCare in Wisconsin $[8,16]$ were early adopters of Lean and have demonstrated the effectiveness of a Lean approach to heath system management. At Seattle Children's Hospital (SCH), Lean-based continuous performance improvement (CPI) has been an integral part of daily operations and management for over a decade $[17,18]$. In this article, we will briefly put Lean into context as it relates to other improvement frameworks, introduce Lean concepts through a "Lean Primer," and provide descriptive examples of how Lean has been applied in the Seattle Children's Hospital Emergency Department to improve our system of care. We conclude with reflections and lessons learned through our experience.

\section{Putting Lean into context}

Several different quality improvement frameworks share the goal of improving quality and efficiency. They overlap significantly and share a common history using principles from early quality improvement experts such as W. Edwards Deming, Joseph Juran [19], and Walter Shewhart [20]. They differ in which elements of health care processes are the emphasis of achieving their shared goal. Grossly oversimplified, Lean focuses on elimination of waste, Six-sigma attempts to control variation [21], Theory of Constraints targets processes that

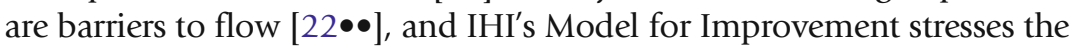

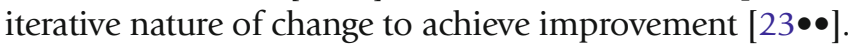

\section{A Lean primer: basic principles, tools, and methods}

\author{
The value stream
}

The complexity of the health care system is perhaps best understood from the point of view of the patient. While care providers may view the system through the lens of their usual environment, a patient's interaction with the system is not isolated to their clinic visit or time in the operating room. Rather, it spans multiple transitions from inpatient to outpatient or from the operating room to the surgical unit. Value stream mapping looks at the totality of the patient's interaction with the health care system. A useful way to understand this concept 
is to actually map each step in the process of a patient who presents for medical care-from arrival until their care is completed-all from the patient's per-

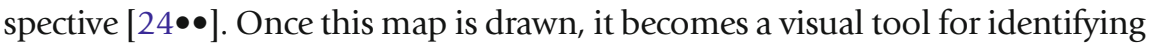
the non-value-added (NVA) or wasteful steps or events a patient may experience. These NVA steps are identified as opportunities for improvement. Thus, the value stream map is the tool that starts the improvement process (Fig 1).

\section{Waste}

At its core, Lean strives to remove waste from a process. Waste is defined as any step which does not provide value to the patient and family (NVA). Valueadded (VA) steps include any step that directly contributes to improvement of the patient's medical condition or experience; another way of defining a VA step is one that the patient would be willing to pay for on an itemized bill [25]. Nonvalue-added (NVA) steps are sometimes necessary because of regulation or safety considerations. The goal of Lean-based process improvement is to eliminate unnecessary NVA steps, reduce necessary NVA time, and improve VA steps. Using these criteria, it has been estimated that $90-95 \%$ of a typical process is waste [26•] and waste in the health care system has been estimated to cost as

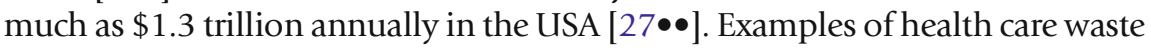
are further defined in Table 1.

A key Lean principle is respect for the people who do the work. Therefore, in any Lean improvement project, it is essential to include front line staff from multiple disciplines and for leadership to go to the actual site of the work (Gemba, the workplace). Bringing the entire improvement team to the workplace enables methodical mapping of the process they seek to improve using Lean tools. One example of a Lean tool is a spaghetti diagram, which maps staff and patient movement. $5 \mathrm{~S}$ is another tool that is used to reduce search time for materials (Table 2).

\section{Standard work}

The ultimate goal for improvement of a process is to achieve flow. In the ideal state, the patient's experience moves seamlessly from one step to the next with

Simple Laceration Repair Process -Steps Include Waiting Time

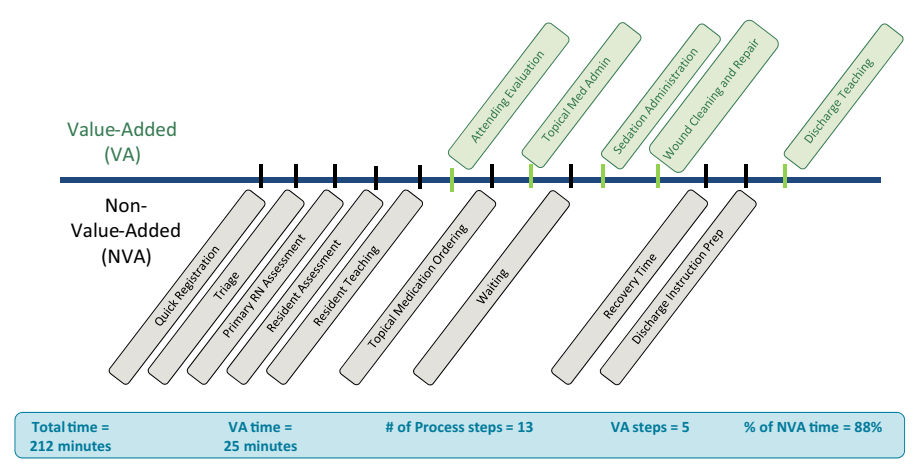

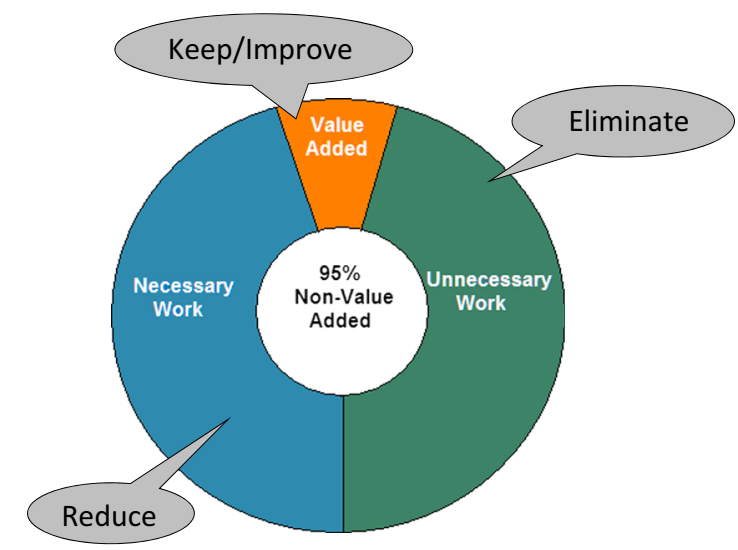

Fig. 1. Value stream/process map. 
Table 1. Examples of health care waste

\begin{tabular}{|c|c|}
\hline Potential health care waste & Description/example \\
\hline Processing & Asking patients the same questions multiple times \\
\hline Correction (i.e., rework) & Medical errors \\
\hline Searching & Information, materials, staff, and equipment are not readily available \\
\hline Transportation & Any unnecessary movement of staff, goods, or patients \\
\hline Underutilized staff & Unbalanced work causing some staff to be overburdened and others to be underused \\
\hline Inventory & $\begin{array}{l}\text { Differing supply needs for the same procedure based on provider preference; excess supply } \\
\text { can lead to expired items and increase search time }\end{array}$ \\
\hline Complexity & $\begin{array}{l}\text { Excess paperwork; differing and unpredictable treatment paths based solely on provider } \\
\text { preference }\end{array}$ \\
\hline Waiting & A symptom of all other types of waste \\
\hline
\end{tabular}

minimal waste and no barriers. In this state, everyone knows what to expect next and the amount of time spent by the patient in the health care setting is reduced to the time necessary to safely and effectively treat their medical condition. This state cannot be achieved instantly. One prerequisite for flow is to standardize processes wherever possible. Without standardization, it is impossible to identify the foundation upon which to apply iterative cycles of improvement. When standard work is accepted and followed by staff, each step in a process becomes more predictable and time-based which allows the opportunity for a data-driven review of changes. When time can be attributed to a reliably followed process step, it is called a reliable method. Variation in medicine cannot be completely eliminated but should be driven by the unique needs of patients rather than preferences of the staff.

\section{The "Pull" system}

When a process cannot yet flow efficiently, an interim step is to achieve a state of pull. In a pull system, there is a signal, or kanban, which triggers movement of the patient or item to the next step in the process. The patient does not move until the next step is ready. This one-piece flow is much more efficient than traditional batch processing. However, it is important to emphasize that developing a pull system is not the ultimate goal. A pull system without equally balanced work causes waiting between steps. The goal of a pull system is to identify where constraints exist to allow iterative improvement to remove them. In an ideally developing Lean process, each improvement effort identifies the next process that needs improvement. As with IHI's model for improvement,

\section{Table 2. Definitions and descriptions of $5 \mathrm{~S}$}

\section{$5 S$}

Sort

Straighten

Scrub

Standardize

Sustain

\section{Description}

Separate unneeded from needed A place for everything and everything in its place Visual and physical inspection

Everyone doing the same thing Hold the gains through audits and accountability 
this requires a commitment to continuous plan-do-study-act (PDSA) cycles. This commitment to continuous improvement is called kaizen and is much easier to sustain when improvement is built into the daily work of front line staff and their supervisors.

\section{Built-in quality}

An intended by-product of one-piece flow is attention to quality and "error proofing" processes whenever possible. In an ideal one-piece flow state, each step in the process is able to immediately identify and rectify any errors or problems without the patient moving to subsequent steps, where problems are much more difficult to identify and understand. In the ideal setting, work stops as soon as a problem is noticed and that problem is addressed immediately. In the Toyota production system, this concept is called jidoka.

\section{Rapid process improvement workshop}

A Rapid Process Improvement Workshop (RPIW) is a Lean-based tool used to develop and implement process changes. The goal of the RPIW is to pull together a multidisciplinary team (including key executives, faculty leaders, front line staff, and patient/family representatives) who work together to redesign and implement a process change in 5 days time. The scope of the workshop is clearly outlined in a charter document, and there is an RPIW executive sponsor who is responsible for keeping the workshop on track and removing logistic or cultural obstacles. RPIW teams are expected to develop proposed design changes and share them with stakeholders during the workshop week. The new design is implemented at the end of the week and is audited in a series of Plan-Do-Check-Act (PDCA, the Lean version of PDSA) cycles at 30, 60, and 90 days.

\section{Lean in the emergency department}

Numerous studies have described the implementation of Lean methodologies in the emergency department (ED) setting $[3,28,29]$. Specifically, Lean methodologies have been utilized in the ED to redesign processes [30, $31-35]$, improve patient access and flow [36-47], reduce wait times [48-50], and to address issues such as errors and ED overcrowding [51]. Our institution has applied Lean methodologies to numerous improvement projects, impacting both clinical and operational practice, summarized in Table 3. We describe 3 examples from our experience at Seattle Children's Hospital (SCH) in greater detail below.

\section{Seattle Children's Hospital ED front-end model redesign [30॰]}

A 26-member multidisciplinary team met over 5 days to redesign our front-end ED care team structure and workflow using Lean methodologies (RPIW). The goal of the redesign process was to create a new model that reduced time to be seen by a licensed ordering provider to $30 \mathrm{~min}$ or less for $80 \%$ of patients. Lean design principles included careful observation of current state processes, analysis of each step in the process to determine if they are completely unnecessary, unnecessary but unavoidable, or necessary (value-added) (i.e., Value Stream 


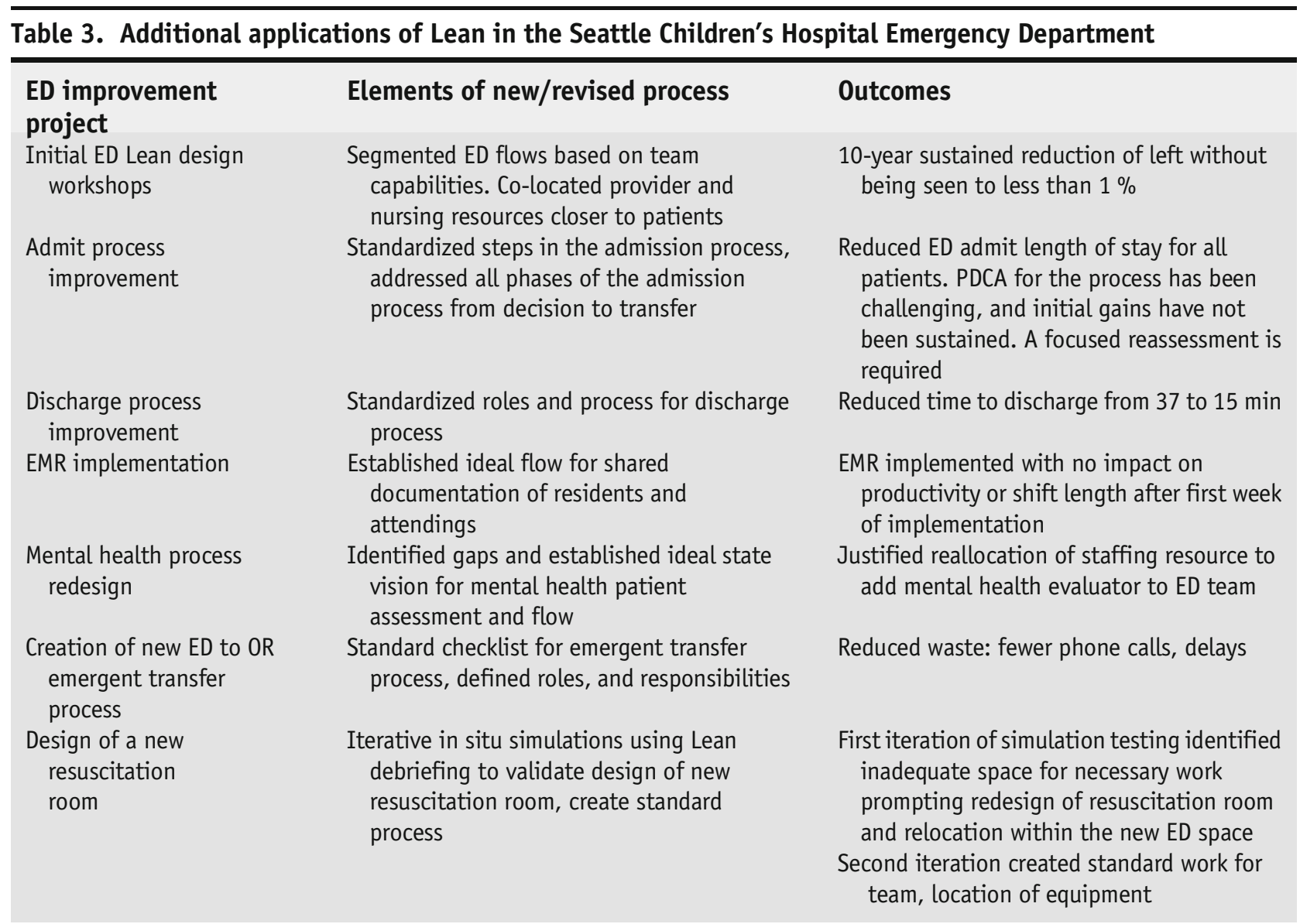

Mapping). Based on a review of ED front-end literature, mapping of the current system through direct observation, and methodical elimination of non-valueadded steps, a new model was designed. In our "current state," we used a traditional front-end model found in many academic emergency departments. Current state patient flow was mapped out as follows: On arrival, patients were greeted by security and administrative staff in the waiting room. Next, they saw the triage nurse who obtained vital signs and assigned a triage level. If a room was not immediately available, patients were sent to the waiting room after triage. Once roomed, patients were seen by registration staff, medication intake coordinators (trained pharmacy technicians who verify and enter all home medications, dose, and frequency into the computer), nurses and multiple physicians or trainees including medical students, residents, fellows, and attendings in a series of often redundant encounters.

NVA steps identified in this model included delay from patient arrival to provider, patient wait times to nursing assessment, travel times, redundant questioning about medications and historical information, and providers not always available to see patients when they arrive to a patient room. To minimize or eliminate these non-value-added steps, components of previously described front-end models were adopted. 
Patient flow through the new front-end model is as follows: On arrival, patients are first seen by a lobby nurse who does a rapid visual assessment which includes assessing the patient's level of consciousness, work of breathing, and color. The lobby nurse then performs a quick registration which involves entering name, date of birth, and whether an interpreter is needed into the electronic medical record (EMR). Nurses are geographically zoned in the ED and have an electronic mechanism for visually indicating on the tracking system when they are ready for a new patient (Pull system). Medication intake coordinators (MICs) then transport patients (minimizing underutilized staff and transport time) to an open room, where they are greeted by a nurse who obtains vital signs, thereby eliminating traditional triage for most patients. Medication intake is completed by the MIC simultaneously with vital signs. In our ideal state, the medical provider care team is also present to assess the patient once they are placed in a room. The care team listens to the presenting complaint, performs a focused physical exam, and verbalizes an initial plan to the nurse and family. If a physician care team is not available, a separate "Early Initiation" team (physician or NP plus an RN) is available to meet and assess patients, place orders, and start treatments.

The new model was tested over two pilot periods and compared to a similar period of control days. ED census and patient acuity were similar during both pilot periods. Eighteen patients were included in pilot 1, and 80 patients were included in the expanded second pilot. Patients seen within 30 min improved from a baseline of 33 to $93 \%$ in pilot 2 . Time to a visual assessment by a nurse, to a room, and to a licensed independent provider all decreased. The largest decrease was in median time to provider, from $43 \mathrm{~min}$ in the current state to 7 min during pilot 2 .

\section{SCH ED asthma clinical standard work}

Asthma is the most common chronic illness in children and accounts for $>600,000$ ED visits each year. At our institution, asthma is the leading ED diagnosis resulting in hospital admission. We recognized that reducing ED length of stay for moderate to severe asthmatics would improve ED throughput (flow) and patient care for this high risk population. Our institution has used the Respiratory Clinical Score (CS) since 2002 to guide treatment on our standardized clinical pathway for asthma (Fig. 2) [52]. Documentation of respiratory scores before and after treatments allowed a review of asthma patients presenting to our pediatric ED over a 7-month period in 2010-2011. We reviewed respiratory score on arrival to the ED, score after the first and second hours of treatment, disposition (admit/discharge), and ED length of stay for all patients with a diagnosis of asthma who presented to the ED from October 2010 to April 2011. This analysis revealed that $90 \%$ of patients with high scores (9-12) after 1-h of treatment were ultimately admitted to the hospital; however, these patients had variable and often prolonged lengths of stay in the ED prior to admission (NVA step). In order to expedite care and improve ED efficiency, standardized respiratory score-based admission criteria were added to our asthma clinical pathway in September 2011. Mean ED length of stay and time to bed request for admitted asthmatics both decreased by 30 min after implementing the modified asthma pathway with standardized admission criteria. Thus, by standardizing care for asthma patients to include 


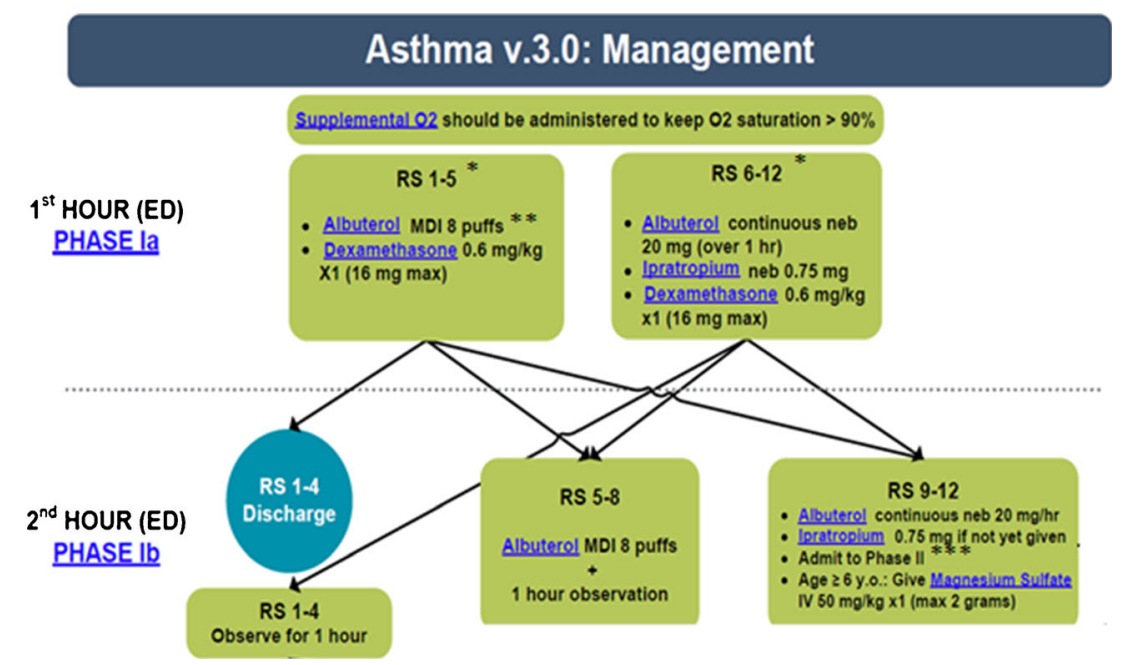

Fig. 2. Example of Asthma Clinical Standard Work. (Asterisk) RS respiratory score, (double asterisk) MDI metered dose inhaler, (triple asterisk) Admit to Phase II standardized admission criteria added to pathway for patients with respiratory score of 9-12 after the first hour of treatment.

objective admission criteria early in the ED course, we optimized patient care and improve ED flow.

Lean-focused simulation and the SCH ED resuscitation room redesign (5S)

Our institution has integrated Lean methodology with simulation methodology. In situ simulation is used to go to gemba for rare or complex processes, identify waste and constraints, design standard work, and perform an initial PDCA cycle, even before rolling out a new process. We have created a simulation process that incorporates Lean tools and a Lean-focused debrief. The goal of Lean-focused simulation is to efficiently identify and address systems and quality issues.

A 13-member multidisciplinary team that included physicians, nurses, and facilities personnel met over 2 days to redesign an existing resuscitation room through the use of in situ simulation and Lean methodologies. The goal of the redesign process was to reduce search and set-up time for materials and remove waste in the process of administering resuscitative care. Two in situ simulations, an infant in cardiogenic shock and a toddler with multiple traumatic injuries, were conducted at the beginning of the 2-day event to identify current state and again at the end of the event to identify changes. At the start of these simulations, participants were oriented to the systems focus of the simulation and provided with the medical case scenario as well as specific targets for the team (e.g., defibrillation of the infant when in pulseless ventricular tachycardia (VTach)). This type of participant orientation is unique in Lean or systems simulation and takes the focus off the medical care itself to allow emphasis on the system and process of delivering care. Lean-focused debriefing was employed to systematically identify waste and to identify opportunities to streamline care. To aid participants in focusing on waste, a poster with the types of waste and examples of each were available for reference. Lean-focused debriefing is different from traditional simulation debriefing in that instead of focusing on the medical knowledge, technical skills, or teamwork of the team; the debrief focus is again 
on the system and process. One example of Lean-focused debriefing from this event involved the time it took the nurse to place an IV in the simulated patient. In the "before" simulation, we identified that it took just over 3 min to place an IV in a critically ill patient, longer than the pediatric advanced life support (PALS) goal of $90 \mathrm{~s}$. An open-ended debriefing question allowed identification of waste in this process, "I noticed that it took over $3 \mathrm{~min}$ to obtain an IV in this patient. What prevented you from being able to place the IV sooner?" The nurse was then able to identify several areas of waste including the need to gather supplies from several drawers to both place an IV and obtain requested labs. In addition to the identification of waste in the delivery of resuscitative care, the event team conducted a $5 \mathrm{~S}$ exercise of the entire room and its contents.

A total of 42 types of waste were identified in areas of inventory, transportation, and motion. Examples of waste included unnecessary duplication of medications in both the code cart and in-room supply dispensing station; the need for the nurse to leave the room to obtain critical resuscitative medications only located in the central ED medication room; the lack of standard IV set-up to allow efficient access to needed IV and lab supplies as discussed in the debriefing example above; and the lack of team role clarity resulting in role confusion and care delays. Using the time allotted, the event team was able to implement several immediate changes including the removal of duplicate medications and inventory which allowed the removal of a large in-room supply dispensing station, thus reducing clutter, establish standard patient bed and IV set-ups, and define standard code team roles. Additional changes were implemented over the next 6-8 weeks including a new in-room locked medication area to allow all resuscitative medications to be in the same location and the standardization of room set up and procedure carts.

The $5 \mathrm{~S}$ exercise resulted in several additional improvements including decreased par level of respiratory supplies in the room (sort); identification of a step stool on the patient's right side for compressions (straighten); removal of an asthma algorithm from 1998 (scrub); clarified code team role locations around the patient (standardize); and creation of a room readiness checklist (sustain) (Fig. 3).
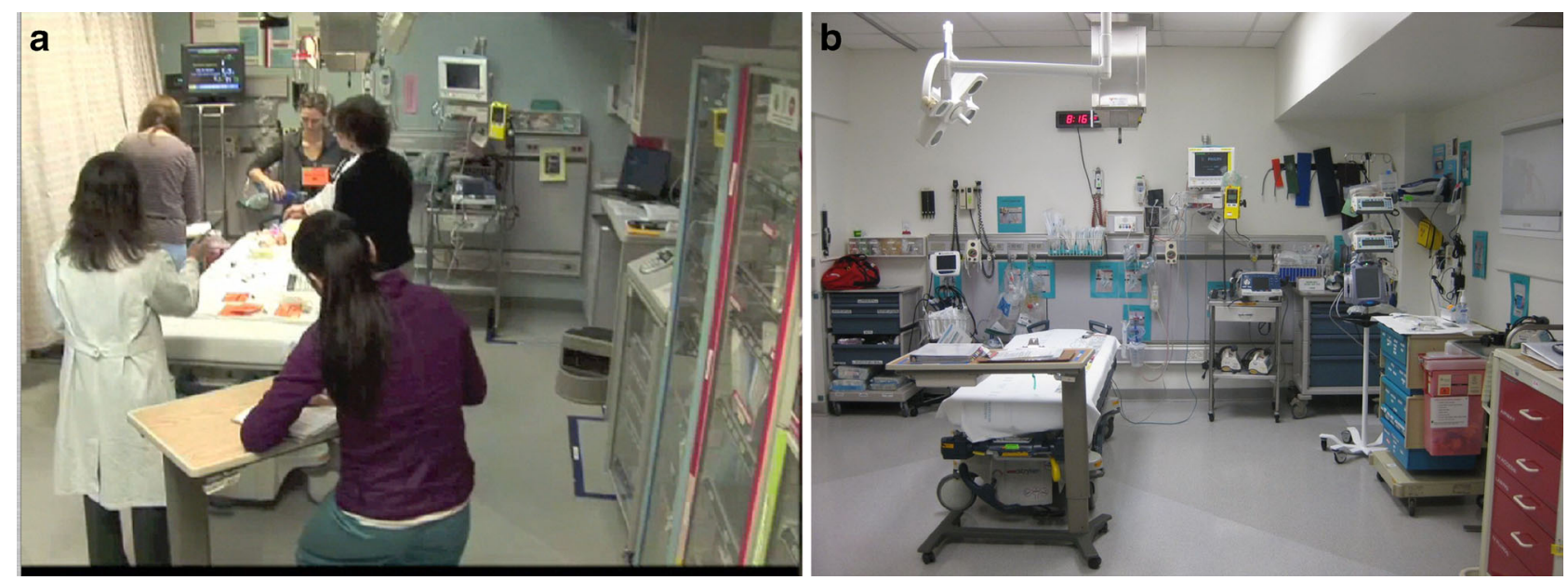

Fig. 3. a ED resuscitation room before Lean-focused redesign event. b ED resuscitation room after Lean-focused redesign event*. *General storage cabinetry eliminated, task-based mobile carts implemented, co-location of supplies based on users (e.g., nursing supplies grouped together). 
Simulations conducted at the end of the event identified overall improvement in the delivery of resuscitative care. Specifically, in the before state, IV access took over 3 min and the team was not able to deliver the first shock for pulseless VTach in the desired $1 \mathrm{~min}$. Following the changes, IV access was obtained in the desired $<90 \mathrm{~s}$ and the first shock for pulseless VTach was delivered in $<1 \mathrm{~min}$. In addition, the team identified that the resuscitation room was less cluttered allowing for the easier identification of necessary equipment and that the provision of resuscitative care was overall more efficient.

\section{Conclusion}

Lean methodology has proved to be an invaluable tool at Seattle Children's Hospital with regards to emergency department operations. As described above, it is an iterative process that may provide short-term successes but should be looked at as a long-term investment. Developing a culture of change acceptance and excitement for the process are the first hurdles. Many workers are comfortable with the status quo, and change is potentially viewed as challenging and disruptive. This can be especially difficult in the emergency department, where many pride themselves on their unique abilities to adapt, go with the flow, and provide care in spite of any obstacles. Lean does not try to remove any of the innovative and entrepreneurial spirit of ED providers but attempts to enable to providers and staff to work at their best without the constraints that we may have accepted as immovable. An example of placing tongue blades and ear speculums next to the otoscope, ensuring these items are always stocked in the area of use is a simplistic but illustrative example of Lean in action. If a provider sees 20 patients on a shift and does not have to look for these objects, or cross the room for supplies each time, they can save a couple minutes per encounter. Multiple that by 20 patients and one has potentially captured an additional $30 \mathrm{~min}$ or more of time that adds value to patients enables greater throughput and increases ED capacity. The risk of Lean, however, is that changes like those noted above with the tongue blades are short-term improvements, and without constant, reliable, and planned PDCA efforts, the change effects may be diminished, or the workers may revert back to the comfort of their older, standard procedures. Garnering enthusiasm and excitement for large change is often fun and rewarding, but the true value in the process is not only making the change(s) suggested by the workers, objective observations, and data but ensuring that the improvement efforts are continually revaluated. These may be affected by other changes, or the focus may shift when other new and exciting ideas/changes are brought forward.

As with any improvement process, the initial change is valuable, but the long-term impact is determined by maintaining focus and critical review. Teams also need to be cognizant of unintended consequences. Improvements in one area may uncover issues in others that need to be addressed or managed. These changes will then, in turn effect other procedures. Most change cannot be looked at in isolation but needs to be viewed as part of the larger environmental milieu. As suggested above, support for staff who may be complacent or openly antagonistic/disruptive need to be available. While the change needs to be from the workers up, management of the staff and a commitment to the process and improvement needs to be from the leadership. Presence, enthusiasm, patience, 
and respect for the process and people are vital components that need to be present in Lean leaders. One aspect that has lead to the successful implementation of Lean at Seattle Children's Hospital is that both the hospital and medical leadership are all strong supporters of the methodology, speak the same process improvement language, and are able to generate support and resources for operation-wide forward movement. Attempting to make substantial change as an isolated division, department, or discipline is very difficult and likely not sustainable. Also, as noted, involvement of families and patients can be invaluable. They are indeed the customers for our processes and can help ground us in our approach and goals. We are privileged to have a strong parent/ family council who have members involved in almost every aspect of our operation and serve to make us better on a daily basis.

\section{Compliance with Ethical Standards}

\section{Conflict of Interest}

Lori Rutman declares that she has no conflict of interest. Kimberly Stone declares that she has no conflict of interest. Jennifer Reid declares that she has no conflict of interest. George A (Tony) Woodward declares that he has no conflict of interest. Russell Migita declares that he has no conflict of interest.

\section{Human and Animal Rights and Informed Consent}

This article does not contain any studies with human or animal subjects performed by any of the authors.

\section{References and Recommended Reading}

Papers of particular interest, published recently, have been highlighted as:

- Of importance

$\bullet \quad$ Of major importance

1. Ohno T, Bodek N. Toyota production system: beyond large scale production. New York: Productivity Press; 1988.

2. Deming WE. The new economics. 2nd ed. Cambridge: Center for Advanced Educational Services, MIT; 1994.

3. Holden RJ. Lean thinking in emergency departments: a critical review. Ann Emerg Med. 2011;57:265-78.

4. Womack JP, Jones DT, Roos D. The machine that changed the world. New York: Free Press; 2007.

5. Jones D, Mitchell A. Lean thinking for the NHS. London: NHS Confederation; 2006.

6. Institute of Medicine. Best care at lower cost: the path to continuously learning health care in America.

Washington DC: National Academies Press; 2012.

7. Spear SJ. Fixing health care from the inside, today. Harv Bus Rev. 2005;83:78-91.

8. Toussaint J. Writing the new playbook for US healthcare: lessons from Wisconsin. Health Aff. 2009;28:1343-50.
9. Nelson-Peterson DL, Leppa CJ. Creating an environment for caring using Lean principles of the Virginia Mason Production System. J Nurs Adm. 2007;37:287-94.

10. Fillingham D. Lean healthcare: improving the patient's experience. Chichester: Kingsham; 2008.

11. Cooper RG, Mohabeersingh C. Lean thinking for medical practices. J Pre-clin Clin Res. 2008;2:88-9.

12. Ben-Tovim DI. Letters. Seeing the picture through "lean thinking”. BMJ. 2007;334:169.

13. Miller D. Going lean in health care. Cambridge: Institute for Healthcare Improvement; 2005.

14. Bush RW. Reducing waste in US health care systems. JAMA. 2007;297:871-4.

15. Kenney C. Transforming health care: Virginia Mason Medical Center's pursuit of the perfect patient experience. New York: Productivity Press; 2010.

16. Toussaint JS, Gerard R. On the mend: revolutionizing healthcare to save lives and transform the industry. Cambridge: Lean Enterprise Institute; 2010. 
17. Wellman J, Hagan P, Jeffries H. Leading the lean healthcare journey: driving culture change to increase value. New York: Productivity Press; 2011.

18. Stapleton FB, Hendricks J, Hagan P, Del Beccaro M. Modifying the Toyota production system for continuous performance improvement in an academic children's hospital. Pediatr Clin N Am. 2009;56:799-813.

19. Juran J. Quality control handbook. 3rd ed. New York: McGraw-Hill; 1979.

20. Shewhart WA. Economic control of quality of manufactured product. New York: D. Van Nostrand Company; 1931.

21. DelliFraine JL, Langabeer II JR, Nembhard IM. Assessing the evidence of six sigma and lean in the health care industry. Qual Manag Health Care. 2010;19(3):211-25.

22.• Goldratt EM, Cox J. The goal: a process of ongoing improvement. Great Barrington: North River Press; 2014.

This book is an easy and engaging read and provides a foundational understanding of the Theory of Constraints.

23.• Scoville R, Little K. Comparing lean and quality improvement. IHI white paper. Cambridge: Institute for Healthcare Improvement; 2014. (available at ihi.org).

This White Paper from the IHI provides a comprehensive and insightful comparison of lean and other quality improvement frameworks that may be applied in healthcare.

$24 . \bullet$ Toussaint JS, Berry LL. The promise of lean in health care. Mayo Clin Proc. 2013;88(1):74-82.

This article provides an overview of Lean principles with applications to health care using examples from health care systems that have implemented Lean.

25. Wellman J. Excerpt from "waste module: lean leader training/rapid process improvement" copyright. Joan Wellman \& Associates, Inc: Seattle; 2005.

26. Hagan P. Waste not, want not: leading the Lean healthcare journey at Seattle Children's Hospital. Glob Bus Organ Excell. 2011;30:25-31.

This case study from then president and COO, Pat Hagan, provides an overview of Seattle Children's Hospital experience in implementing Lean including the incremental approach, lessons learned from observations in Japan and outcome examples.

27.•• Berwick DM, Hackbarth AD. Eliminating waste in US health care. JAMA. 2012;307:1513-6.

This paper defines waste within the current US healthcare system, estimates the cost of each type of healthcare waste, and provides an important perspective on the opportunities for waste reduction during this time of healthcare reform.

28. Dickson EW, Singh S, Cheung DS, Wyatt CC, Nugent AS. Application of lean manufacturing techniques in the emergency department. J Emerg Med. 2009;37(2):177-82.

29. Dickson EW, Anguelov Z, Vetterick D, et al. Use of Lean in the emergency department: a case series of 4 hospitals. Ann Emerg Med. 2009;54:504-10.

30. Rutman LE, Migita R, Woodward GA, et al. Creating a leaner pediatric emergency department: how rapid design and testing of a front-end model led to decreased wait time. Pediatr Emerg Care.

2015;31(6):395-8.

This paper describes the process our group used to develop and pilot test a new front-end model in real time in a busy pediatric emergency department before full implementation in our new facility.

31. Ben-Tovim DI, Bassham JE, Bolch D, et al. Lean thinking across a hospital: redesigning care at the Flinders Medical Centre. Aust Health Rev. 2007;31:10-5.

32. Ben-Tovim DI, Bassham JE, Bennett DM, et al. Redesigning care at the Flinders Medical Centre: clinical process redesign using "lean thinking". Med J Aust. 2008;188:S27-31.

33. Jacobson GH, McCoin NS, Lescallette R, et al. Kaizen: a method of process improvement in the emergency department. Acad Emerg Med. 2009;16:1341-9.

34. Retallick N. Patient process improvements in the emergency department [abstract]. Australas Emerg Nurs J. 2007;10:221-2.

35. Schuur JD, Collins D, Smith A, et al. Use of Lean techniques to simplify admission procedures and decreased ED process time [abstract]. Ann Emerg Med. 2007;50:S90.

36. Kaale RL, Vega DD, Messner K, et al. Time value stream mapping as a tool to measure patient flow through emergency department triage [abstract]. Ann Emerg Med. 2005;46:S108.

37. Kelly A-M, Bryant M, Cox L, et al. Improving emergency department efficiency by patient streaming to outcomes-based teams. Aust Health Rev. 2007;31:16-21.

38. MacLeod H, Bell B, Deane K, et al. Creating sustained improvements in patient access and flow: experiences from three Ontario healthcare institutions. Healthc Qual. 2008;11:38-49.

39. King DL, Ben-Tovim DI, Bassham J. Redesigning emergency department patient flows: application of Lean thinking to health care. Emerg Med Australas. 2006;18:391-7.

40. Dickson EEW, Anguelov Z, Bott P, et al. The sustainable improvement of patient flow in an emergency treatment centre using Lean. Int J Six Sigma Compet Adv. 2008;4:289-304.

41. Eller A. Rapid assessment and disposition: applying Lean in the emergency department. J Healthc Qual. 2009;31:17-22.

42. Woodward GA, Godt MG, Fisher K, et al. Children's hospital and regional medical center emergency department patient flow-rapid process improvement (RPI). In: Chalice R, editor. Improving healthcare quality using Toyota lean production methods: 46 steps for improvement. 2nd ed. Milwaukee: Quality; 2007. p. 145-50.

43. Ieraci S, Digiusto E, Sonntag P, et al. Streaming by case complexity: evaluation of a model for emergency department fast track. Emerg Med Australas. 2008;20:241-9. 
44. Kelly A-M, Bryant M, Cox L. Initiative in redesigning emergency care to improve patient flow [abstract]. Australas Emerg Nurs J. 2007;10:226.

45. O'Brien D, Williams A, Blondell $\mathrm{K}$, et al. Impact of streaming "fast track" emergency department patients Aust Health Rev. 2006;30:525-32.

46. Massucci JL, Farley H, Laskowski Jones L, et al. Reduction in emergency department fast track length of stay [abstract]. Ann Emerg Med. 2008;52:S111.

47. Migita R, Del Beccaro M, Cotter D, Woodward GA. Emergency department overcrowding: developing emergency department capacity through process improvement. Clin Pediatr Emerg Med. 2011;12(2):141-50.
48. Ng D, Vail G, Thomas S, et al. Applying Lean principles of the Toyota Production System to reduce wait times in the emergency department. CJEM. 2010;12:50-7.

49. Parks JK, Klein J, Frankel HL, Friese RS, Shafi S. Dissecting delays in trauma care using corporate lean six sigma methodology. J Trauma. 2008;65(6):1098-104.

50. Weinstock M, Storyboard. How one hospital slashed ED waits. Hosp Health Netw. 2007;81(12):22-30.

51. Kulkarni RG. A reader and author respond to "going lean in the emergency department: a strategy for addressing emergency department overcrowding". Medscape J Med. 2008;10:25.

52. Liu LL, Gallaher MM, Davis RL, et al. Use of a respiratory clinical score among different providers. Pediatr Pulmonol. 2004;34:243-8. 